\title{
The dynamics of the floodwater and the damaged ship in waves
}

\author{
Zhiliang Gao (高志亮) ${ }^{1 *}$, Dracos Vassalos ${ }^{2}$ \\ ${ }^{1}$ Research Center of Coastal and Estuarine Engineering, Tianjin Research Institute for Water Transport \\ Engineering, M.O.T., Tianjin, 300456, China \\ ${ }^{2}$ Department of Naval Architecture and Marine Engineering, University of Strathclyde, \\ Glasgow, G4 0LZ, United Kingdom \\ *E-mail: zlgao@hotmail.com
}

\begin{abstract}
The interaction between the dynamics of the floodwater and the damaged ship in waves is investigated by applying an integrated method, which couples a seakeeping solver and a Navier-Stokes solver. To reveal the effects of the water flooding and the sloshing on the damaged ship behaviour, the motion of a Ro-Ro ferry in regular beam seas is simulated, including the ship under the intact condition with and without internal water and the ship under a damaged condition. It is found that the shift of the natural roll frequency of a damaged ship and the decrease of its roll response are mainly due to the water sloshing inside the compartment. The effect of the resonant sloshing leads to the presence of a ship's second peak response at higher frequencies and it is significantly reduced by the water flooding through the damaged opening. The influence of the flooding and the sloshing on the ship behaviour is small with a further increase of the wave frequency.
\end{abstract}

Key words: damaged ship; flooding; sloshing; coupled model

\section{Introduction}

The safety of ships under a damaged condition is of a prime interest to the maritime industry, the regulatory institutions and the scientific community. In a series of EU funded projects (e.g., HARDER, NEREUS, ROROPROB, SAFEDOR, FLOODSTAND and GOALDS), considerable research effort has been put in the assessment and improvement of damaged ship's stability and survivability. The ship's behaviour upon flooding is one of the key issues in the research, but its underlying mechanism is still not well understood due to the complicated dynamics between the floodwater and the damaged ship.

At the beginning of the flooding process, the high hydrostatic pressure across the damaged opening drives the external water to flood into the empty compartment drastically. The violent floodwater motion may capsize the ship or make it sink rapidly. After this initial stage, the flooding tends to be quasi-stationary. The water flows in and out through the damaged opening depending on the ship behaviour and the external sea state. These mechanisms are even more complicated if a ship is damaged in waves. On one hand, the ship motion is excited by sea waves and at the same time excites the water sloshing inside the compartment. Only a slight motion is sufficient to trigger a violent sloshing when the excitation frequency is in the vicinity of the first natural frequency of the internal water motion. Conversely, the water sloshing also influences the ship motion. On the other hand, the water flooding is accompanied with the exchange of mass and momentum between the internal and external water, and consequently, the characteristics of the water sloshing and the ship motion are changed. The highly coupled dynamics between the floodwater and the damaged ship makes it hard to model the inherent phenomena reliably.

Over the past three decades, a variety of numerical methods that couple the floodwater and the damaged ship motion were proposed. Because the sea water loads on the external hull surface can be effectively calculated based on the seakeeping theory, the development of numerical methods mainly focused on the modeling of the water motion through the opening and inside the compartment. In the earlier studies [1-3], the modified empirical Bernoulli's equation was used to evaluate the flow rate through the opening. The water surface inside the 
compartment was assumed to be horizontal. Subsequently, the concept of the moving lump mass [4-6], the shallow water equation [7-9], the multimodal method [9] and the state-of-the-art computational fluid dynamics (CFD) method [10-12] were introduced to improve the model of the floodwater motion. Just a few of related references are listed; a comprehensive review of the capacity and the limitation of above-mentioned methods may be found in [13].

With the improvement of numerical methods, some attempts were made to study the hydrodynamics of damaged ships based on numerical simulations. Santos and Guedes Soares [8] investigated the interactive dynamics of the water sloshing, the ship and the sea waves. Although the effect of the water flooding was not taken into account, the peculiar behaviour of the damaged ship was partially explained. Gao and Vassalos [14] analyzed the added inertial and damping coefficients of a damaged ship section in a roll decay motion. In their later study [15], the forced roll motion of the same ship section was simulated. The interaction between the flooding and the sloshing and their effects on the ship hydrodynamics were evaluated.

This paper extends the studies previously conducted by the authors [16], where an integrated numerical method combined with the CFD and the seakeeping theories was developed to simulate the damaged ship flooding in waves. Good agreement between the numerical and experimental results was reported. In this study, the integrated method is employed to simulate three test cases of a Ro-Ro ferry subjected to regular beam seas. The test cases include the ship under the intact condition without internal water, the ship under the intact condition with water sloshing and the ship under a damaged condition with water flooding and sloshing. The floodwater and ship motions in these cases are investigated, in order to better understand the effects of the water flooding and the sloshing on the damaged ship behaviour in waves.

\section{Integrated method}

An integrated method that couples a seakeeping solver and a Navier-Stokes (NS) solver is used for the flooding problem of a damaged ship in this paper. Its mathematical and numerical models were described in detail in [16]. Only a brief introduction of this method is given here.

A seakeeping solver PROTEUS3 [5] is used to calculate the hydrostatic and hydrodynamic forces induced by the sea waves on the external hull surface. The hydrostatic and Froude-Krylov forces are evaluated by integrating the pressure over the instantaneous wetted surface of the ship. The diffraction and radiation forces are first obtained with the strip theory based on the potential flow theory in the frequency domain, and are then transformed into the time domain with spectral and convolution techniques, respectively. To account for the viscous effects outside the ship, the well-known Ikeda's method [17] is employed to correct the roll damping.

A finite-volume-discretisation based NS solver previously developed by the authors $[18,19]$ is used to model the water flooding through the damaged opening and the sloshing inside the compartment. To reduce the computational cost, a sub-region including the damaged openings, the floodable compartments and an external region around the damaged ship section is defined from the entire flow region. The flow motion in this sub-region is computed by solving the unsteady NS equations accompanied with the continuity equation. A VOF family algorithm, CICSAM, proposed by Ubbink and Issa [20], is used to capture the free surface. The ship motion is tackled with the dynamic mesh technique without the turbulence modelling.

At each time step, the boundary condition (e.g., the flow velocity and the water level) of the NS solver is provided by the seakeeping solver. At the same time, the ship motion that excites the floodwater motion inside the compartments is treated as a moving boundary of the NS solver. After the internal liquid load obtained by the NS solver and the sea wave loads obtained by the seakeeping solver are added together, the ship motion at the next time step is updated by solving the linear and angular momentum equations.

\section{Results and discussions}

The previous study [16] demonstrates that the integrated numerical method can reproduce the particular behaviour of a damaged ship in waves (e.g., the shift of the natural roll frequency, the decrease of the peak response and the presence of a second peak response) through a comparison between the numerical and experimental results. To further investigate the effect of the floodwater motion on the ship behaviour, additional numerical tests are carried out using the integrated method in this study. A Ro-Ro ferry for the ITTC benchmarking study [21] is selected as the test ship. Its main specifications and the body plan are shown in Table 1 and Fig.1, respectively. The damaged opening is located on the port side of the midship. Two double bottom tanks and two storage rooms are flooded and their layouts are illustrated in Fig.2. 
Table 1 Main specifications of the ferry

\begin{tabular}{lr}
\hline Length between perpendiculars $\left(L_{\mathrm{pp}}\right)$ & $170.00 \mathrm{~m}$ \\
Breadth $(B)$ & $27.80 \mathrm{~m}$ \\
Draft $(T)$ & $6.25 \mathrm{~m}$ \\
Depth to car deck $(D)$ & $9.00 \mathrm{~m}$ \\
Depth to upper deck $\left(D_{\mathrm{ud}}\right)$ & $17.78 \mathrm{~m}$ \\
Damaged length $\left(L_{\mathrm{dam}}\right)$ & $8.10 \mathrm{~m}$ \\
Centre of gravity above base $(\mathrm{KG})$ & $12.89 \mathrm{~m}$ \\
Displacement $(\Delta)$ & $17,300 \mathrm{t}$ \\
\hline
\end{tabular}

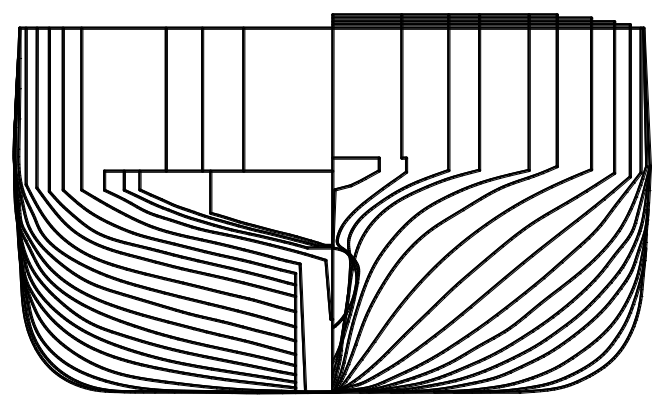

Fig.1 Body plan of the ferry [21]

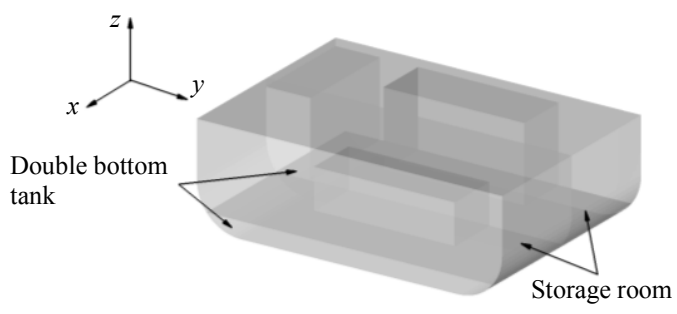

Fig.2 Layout of the floodable compartments

The simulation cases include the ship under the intact condition with and without internal water and the ship under a damaged condition. The wave condition is that in regular beam seas with the wave height of $1.2 \mathrm{~m}$ and the frequency from 0.3 to $1.1 \mathrm{rad} / \mathrm{s}$. In the case of a damaged ship, the CFD simulation domain consisting of the four floodable compartments and a small region outside the compartments is shown in Fig.3. On the top boundary, the pressure is set to be the atmospheric pressure. The hydrostatic pressure corresponding to the initial water height is imposed on the bottom boundary. The elevation and the particle velocity of the incident wave are imposed on the left boundary. On the right, front and back boundaries, the initial water height and the hydrostatic pressure are imposed. The no-slip wall condition is imposed on the surfaces of the hull and the compartments. In the case of the intact ship with internal water, the CFD simulation domain is the floodable compartments. The no-slip wall condition is imposed on the compartment surface. Only four degrees of freedom of the ship (the sway, the heave, the roll and the pitch) are considered in the numerical simulation. In the two cases of the ship with internal water, the floodable compartments are initially filled with water, the level of which is equal to that of the external calm water. The simulation is run on a dual-core (Intel i5, 3.4 GHz) personal computer and continues until the ship motion becomes stable for each wave frequency.

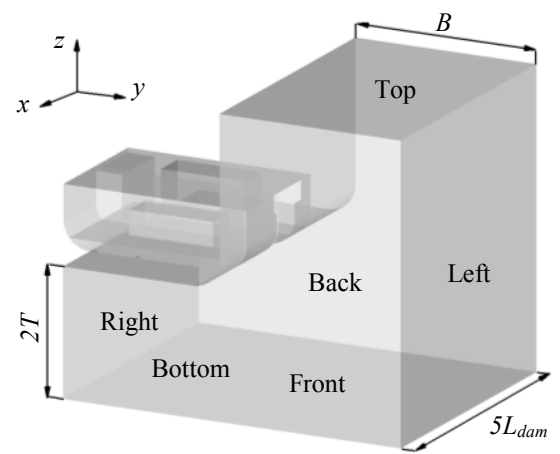

Fig. 3 CFD simulation domain in the damaged ship case 
The water motion inside the compartment significantly influences the ship behaviour. To investigate the grid sensitivity in the case of the internal water motion, three meshes as listed in Table 2 are generated to simulate the the intact ship with internal water. Three wave frequencies of $0.4,0.75$ and $0.9 \mathrm{rad} / \mathrm{s}$ are selected for the grid dependence study. The computational time steps for the coarse, medium and fine grids are $0.02,0.01$ and $0.005 \mathrm{~s}$, respectively. The adopted time step for each grid ensures that the cell Courant number is within the limit of 0.5. It takes approximately 12,63 and $291 \mathrm{CPU}$ hours to finish a 200-s simulation on the coarse, medium and fine grids, respectively. The comparison of the roll response amplitude operators (RAOs) computed with different grid sizes is shown in Table 3. The error between the results obtained on the coarser and finer levels of grid size is less than $2 \%$. Figs. 5 through 7 show the motions of the internal water at the position of four slices illustrated in Fig.4. Small differences of water surface profile obtained with different grid sizes are observed, but the global motions of water as predicted with the three girds are in good agreement. In the following numerical simulations, the medium grid with time step of $0.01 \mathrm{~s}$ is employed for the case of the intact ship with internal water to achieve as high fidelity as possible with acceptable computational cost. In the case of a damaged ship, the mesh arrangement inside the compartments is identical to that of medium grid listed in Table 2. Outside the compartments, the element sizes gradually expand away from the damaged opening. The total number of grid elements is 312,589 . The computational time step is $0.01 \mathrm{~s}$. It takes nearly $117 \mathrm{CPU}$ hours to finish a 200 -s simulation.

Table 2 Meshes employed in the gird dependence study in case of intact ship with internal water

\begin{tabular}{ccccccc}
\hline \multirow{2}{*}{ Grid } & \multicolumn{3}{c}{ Element size $(\mathrm{m})$} & \multicolumn{4}{c}{ Element number } \\
\cline { 2 - 7 } & $\delta x$ & $\delta y$ & $\delta z$ & Double bottom tanks & Storage rooms & Total \\
\hline Coarse & 0.400 & 0.400 & 0.400 & 11,808 & 46,944 & 58,752 \\
Medium & 0.283 & 0.283 & 0.283 & 31,756 & 138,363 & 170,119 \\
Fine & 0.200 & 0.200 & 0.200 & 94,912 & 365,952 & 460,864 \\
\hline
\end{tabular}

Table 3 Comparison of roll RAOs computed with different meshes

\begin{tabular}{cccc}
\hline \multirow{2}{*}{ Grid } & \multicolumn{3}{c}{ Wave frequency $(\mathrm{rad} / \mathrm{s})$} \\
\cline { 2 - 4 } & 0.4 & 0.75 & 0.9 \\
\hline Coarse & 6.44 & 2.86 & 0.67 \\
Medium & 6.52 & 2.83 & 0.66 \\
Fine & 6.58 & 2.85 & 0.66 \\
\hline
\end{tabular}

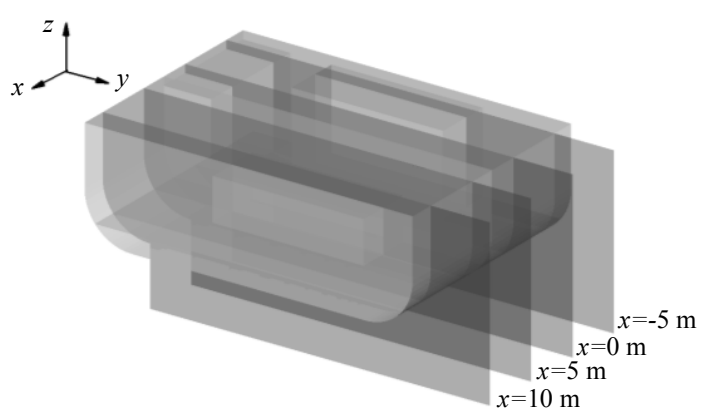

Fig.4 Sketch of the four slices extracted from the compartment domain

Fig. 8 compares the computed roll RAOs in the intact ship case without internal water, the intact ship case with internal water and the damaged ship case at wave frequencies ranging from 0.3 to $1.1 \mathrm{rad} / \mathrm{s}$. For the two cases with internal water, the frequencies of the first peak response (natural roll frequency of the ship) are in good agreement. They are approximately $0.415 \mathrm{rad} / \mathrm{s}$ and are shifted from $0.49 \mathrm{rad} / \mathrm{s}$ in the case without internal water. This comparison demonstrates that the shift of the natural roll frequency of the ship is due to the water motion inside the compartments but has little to do with the water ingress/egress through the damaged opening. On the other hand, the peak magnitudes in the two cases with internal water are nearly 1.5 times smaller than that in the case without internal water, which indicates that the decrease of the ship response is mainly due to the internal water motion. Note that the decrease of the peak response for the damaged ship is slightly larger than that for the intact ship. This suggests that the water ingress/egress further weakens the ship response. As the wave frequency increases, a second peak of the roll response around the frequency of $0.75 \mathrm{rad} / \mathrm{s}$ is observed in the two cases with internal water. The magnitude of this peak for the intact ship is almost three times as large as that for the damaged ship. The large difference between the magnitudes of these two peaks indicates that the water ingress/egress has a significant influence on the internal water motion and consequently on the ship motion in this frequency range. 
For the wave frequency higher than $1.0 \mathrm{rad} / \mathrm{s}$, the ship responses in the three studied cases are close, implying that the influence of the floodwater motion on the ship behaviour becomes small.

The water load on the compartment is related with the internal water motion. Figs. 9 through 11 show the profiles of the water surface inside the compartment in the case of the intact ship with internal water and the case of a damaged ship. At the wave frequency of $0.415 \mathrm{rad} / \mathrm{s}$ (see Fig.9), the elevation of the free surface inside the compartments varies smoothly along the transverse direction; whereas its variation along the longitudinal direction is small. Generally speaking, the profile of the water surface in the case of the intact ship is similar to that in the case of a damaged ship. This comparison indicates that the effect of the water ingress/egress through the opening on the free surface motion of internal water is small. As a result, the frequencies of the ship's first peak response and the response magnitudes in these two cases are close. At the wave frequency of $0.75 \mathrm{rad} / \mathrm{s}$ (see Fig.10), the resonant sloshing phenomenon of internal water is notable. In the case of the intact ship, a large deformation of the water surface is observed along the transverse and longitudinal directions. The sloshing water impacts on the vertical wall of the compartment, climbs up the obstacle and hits the compartment's ceiling. In the case of a damaged ship, it is apparent that the non-linear sloshing effect is much weaker as compared to the case of an intact ship. The comparison implies that the presence of the second peak in the RAO curve is due to the resonant water sloshing inside the compartments. Additionally, the water flooding through the opening largely suppresses the non-linear motion of internal water around the resonant frequency. As the wave frequency further increases to $0.9 \mathrm{rad} / \mathrm{s}$ (see Fig.11), the strongly non-linear sloshing phenomenon disappears. The water surface tends to be flat and horizontal. The free surface profiles of internal water are similar in the two studied cases, suggesting that the flooding effect on the free surface motion inside the compartment becomes insignificant as the wave frequency increases further away from the resonant sloshing frequency.

\section{Conclusions}

The numerical simulation is conducted to study the dynamics of the floodwater and a damaged ship in waves. Three cases of a Ro-Ro ferry, respectively, under the intact condition without internal water, the intact condition with internal water and a damaged condition, are simulated using an integrated numerical method. The interactions among the water flooding, the sloshing and the ship are investigated. Based on the present numerical results, several conclusions can be drawn for the damaged ship flooding with lower compartments. (i) The shift of the natural roll frequency is due to the water sloshing in the compartments but has little to do with the water flooding through the damaged opening. (ii) The decrease of the ship response at its natural roll frequency mainly comes from the internal water sloshing, whereas the contribution of the water flooding is small. (iii) The effect of the resonant sloshing leads to the presence of a ship's second peak response at higher wave frequencies. This effect is largely alleviated by the water flooding through the damaged opening. (iv) As the wave frequency further increases away from the resonant sloshing frequency, the sloshing and flooding effects on the ship behaviour are small.

\section{Acknowledgement}

This work is supported by the Fundamental Research Funds for the Central Public Research Institutes of China (Grant No: TKS130201 and TKS140201) and the Technology Foundation for Selected Overseas Chinese Scholars (granted by Ministry of Human Resources and Social Security of China).

\section{References}

[1] Spouge J. R. The technical investigation of the sinking of the Ro-Ro ferry European Gateway[J]. Transactions of the Royal Institution of Naval Architects, 1986, 128: 49-72.

[2] Dand I. Hydrodynamic aspects of the sinking of the ferry Herald of Free Enterprise[J]. Transactions of the Royal Institution of Naval Architects, 1989, 131: 145-165.

[3] Vassalos D., Turan O. A realistic approach to assessing the damage survivability of passenger ships. Transactions of the Society of Naval Architects and Marine Engineers, 1994, 102: 367-394.

[4] Spanos D., Papanikolaou A. On the stability of fishing vessels with trapped water on deck[J]. Ship Technology Research, 2001, 48 (3): 124-133.

[5] Jasionowski A. An integrated approach to damage ship survivability assessment[D]. Doctoral Thesis, University of Strathclyde, Glasgow, United Kingdom, 2001.

[6] Manderbacka T. L., Matusiak J. E. Ship motions caused by time-varying extra mass on board[C]. Proceedings of the 12th International Ship Stability Workshop, Washington D. C., 2011, 263-269.

[7] Chang B., Blume P. Survivability of damaged Ro-Ro passenger vessels[J]. Ship Technology Research, 1998 ; 45 (3): $105-117$.

[8] Santos T. A., Guedes Soares C. Study of damaged ship motions taking into account floodwater dynamics[J]. Journal of Marine 
Science and Technology, 2008, 13 (3): 291-307.

[9] Kong X., Faltinsen O.M. Coupling between ship motions and flooding of a damaged ship in waves[C]. Proceedings of the 8th International Conference on Hydrodynamics, Nantes, 2008.

[10] Shen L., Vassalos D. Applications of 3D parallel SPH for sloshing and flooding[C]. Proceedings of the 10th International conferences on the Stability of Ships and Ocean Vehicles, Saint Petersburg, 2009, 723-732.

[11] Strasser C., Jasionowski A., Vassalos D. Calculation of time-to-flood of a box-shaped barge by using CFD[C]. Proceedings of the 10th International conferences on the Stability of Ships and Ocean Vehicles, Saint Petersburg, 2009, 733-740.

[12] Zhang A., Cao X., Ming F., Zhang Z. Investigation on a damaged ship model sinking into water based on three dimensional SPH method[J]. Applied Ocean Research, 2013, 42: 24-31.

[13] Gao Z. A hybrid approach to flooding and damaged ship dynamics[D]. Doctoral Thesis, Universities of Glasgow and Strathclyde, Glasgow, United Kingdom, 2012.

[14] Gao Q., Vassalos D. Numerical study of the roll decay of intact and damaged ships[C]. Proceedings of the 12th International Ship Stability Workshop, Washington D. C., 2011, 277-282.

[15] Gao Q., Vassalos D. Numerical study of damage ship hydrodynamics[J]. Ocean Engineering, 2012, 55: 199-205.

[16] Gao Z., Gao Q., Vassalos D. Numerical study of damaged ship flooding in beam seas[J]. Ocean Engineering, $2013,61:$ 77-87.

[17] Ikeda Y. Prediction methods of roll damping of ships and their application to determine optimum stabilization devices[J]. Marine Technology, 2004, 41 (2): 89-93.

[18] Gao Z., Vassalos D., Gao Q. Numerical simulation of water flooding into a damaged vessel's compartment by the volume of fluid method[J]. Ocean Engineering, 2010, 37 (16): 1428-1442.

[19] Gao Z., Gao Q., Vassalos D. Numerical simulation of flooding of a damaged ship[J]. Ocean Engineering, 2011, 38 (14-15): $1649-1662$.

[20] Ubbink O., Issa R. I. A method for capturing sharp fluid interfaces on arbitrary meshes[J]. Journal of Computational Physics, 1999, 153 (1): 26-50.

[21] Papanikolaou A., Spanos D. The 24th ITTC benchmark study on the numerical prediction of damage ship stability in waves preliminary analysis of results' $[\mathrm{C}]$. Proceedings of the 7th International Workshop on Stability and Operational Safety of Ships, Shanghai, 2004.



$\left(\mathrm{a}_{1}\right) x=-5 \mathrm{~m}$

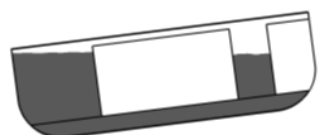

$\left(\mathrm{b}_{1}\right) x=-5 \mathrm{~m}$

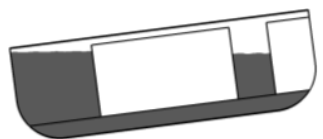

(c $\left.c_{1}\right) x=-5 \mathrm{~m}$

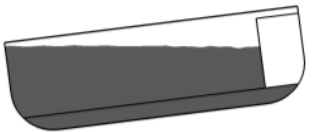

(a $\left.\mathrm{a}_{2}\right) x=0 \mathrm{~m}$

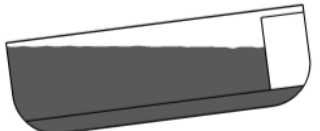

$\left(\mathrm{b}_{2}\right) x=0 \mathrm{~m}$

(b) medium grid

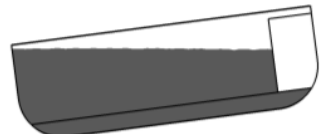

(c $\left.\mathrm{c}_{2}\right) x=0 \mathrm{~m}$

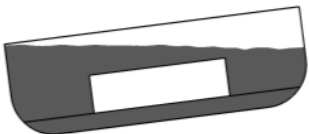

(a) $x=5 \mathrm{~m}$

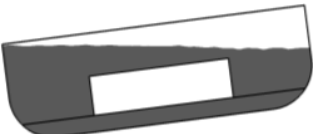

(b $\left.\mathrm{b}_{3}\right) x=5 \mathrm{~m}$

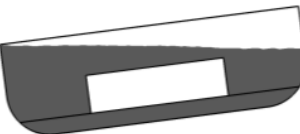

$\left(\mathrm{c}_{3}\right) x=5 \mathrm{~m}$

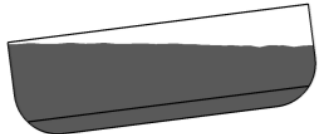

(a) $x=10 \mathrm{~m}$

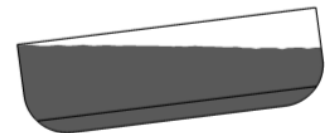

(b $\left.b_{4}\right) x=10 \mathrm{~m}$

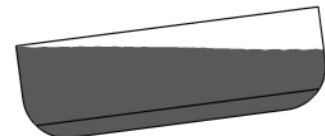

(c) $x=10 \mathrm{~m}$

Fig. 5 Snapshots of internal water motion in the intact ship case at $\omega=0.4 \mathrm{rad} / \mathrm{s}$ and $t=210 \mathrm{~s}$

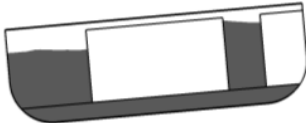

$\left(\mathrm{a}_{1}\right) x=-5 \mathrm{~m}$

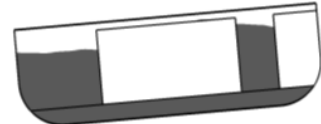

(b $\left.\mathrm{b}_{1}\right)=-5 \mathrm{~m}$

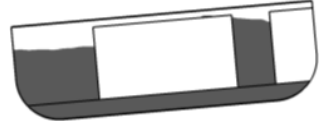

$\left(\mathrm{c}_{1}\right) x=-5 \mathrm{~m}$

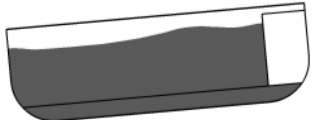

(a $\left.\mathrm{a}_{2}\right)=0 \mathrm{~m}$

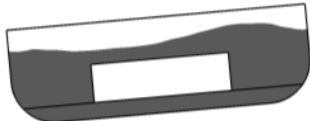

(a) $x=5 \mathrm{~m}$

(a) coarse grid

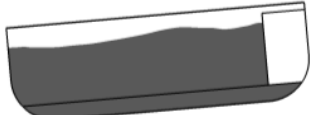

(b $\left.\mathrm{b}_{2}\right)=0 \mathrm{~m}$

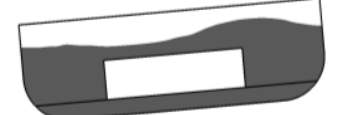

(b) $x=5 \mathrm{~m}$

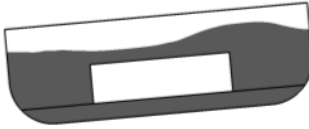

(c) $x=5 \mathrm{~m}$

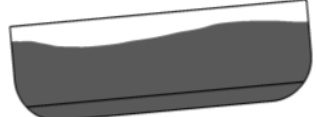

(a) $x=10 \mathrm{~m}$

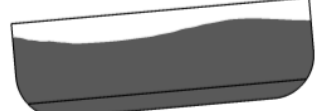

(b $\left.\mathrm{b}_{4}\right)=10 \mathrm{~m}$

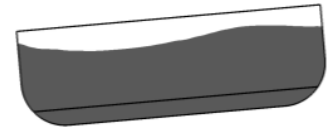

(c $\left.c_{4}\right) x=10 \mathrm{~m}$

Fig.6 Snapshots of internal water motion in the intact ship case at $\omega=0.75 \mathrm{rad} / \mathrm{s}$ and $t=210 \mathrm{~s}$ 


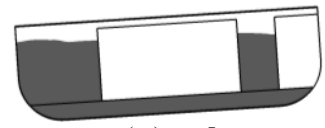

(a $\left.a_{1}\right)=-5 \mathrm{~m}$

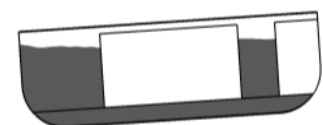

(b 1$) x=-5 \mathrm{~m}$

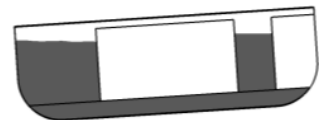

(c $\left.c_{1}\right) x=-5 \mathrm{~m}$

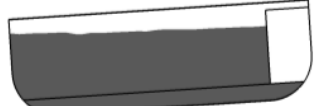

(a $\left.\mathrm{a}_{2}\right)=0 \mathrm{~m}$

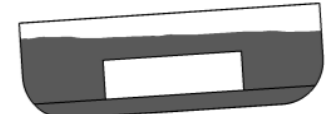

$\left(\mathrm{a}_{3}\right) x=5 \mathrm{~m}$

(a) coarse grid

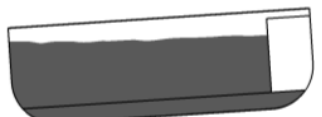

(b) $x=0 \mathrm{~m}$

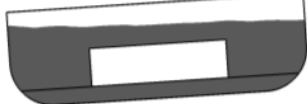

(b $\left.\mathrm{b}_{3}\right)=5 \mathrm{~m}$

(b) medium grid

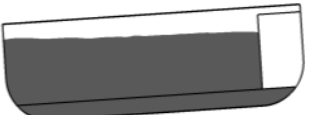

(c $\left.c_{2}\right) x=0 \mathrm{~m}$

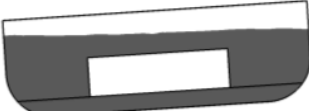

(c $\left.c_{3}\right) x=5 \mathrm{~m}$

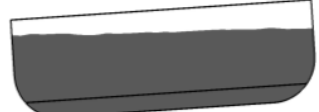

(a) $x=10 \mathrm{~m}$

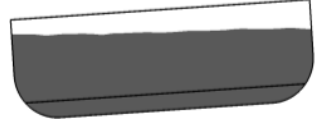

(b4) $x=10 \mathrm{~m}$

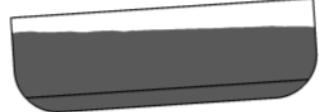

(c) $x=10 \mathrm{~m}$

Fig.7 Snapshots of internal water motion in the intact ship case at $\omega=0.9 \mathrm{rad} / \mathrm{s}$ and $t=210 \mathrm{~s}$

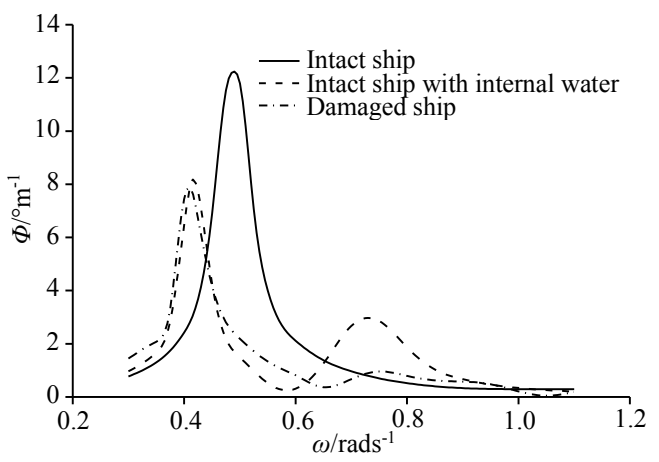

Fig. 8 Comparison of the ship's roll RAO in different cases

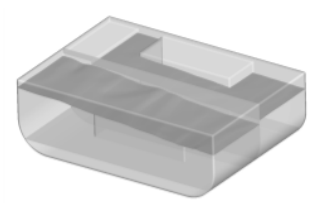

(a 1 ) 0.25 cycle

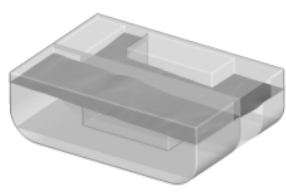

(b $) 0.25$ cycle

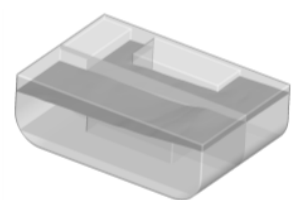

$\left(a_{1}\right) 0.5$ cycle

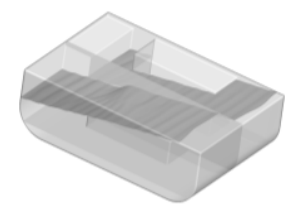

(a $) 0.75$ cycle

(a) intact ship case

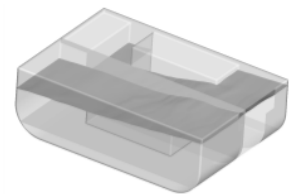

(b $) 0.5$ cycle

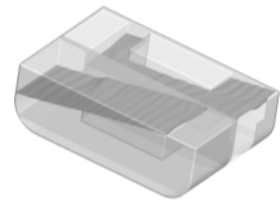

(b) 0.75 cycle

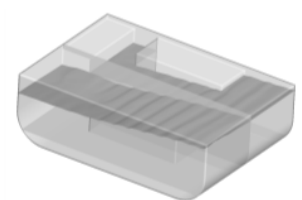

( $\left.\mathrm{a}_{1}\right) 1.0$ cycle

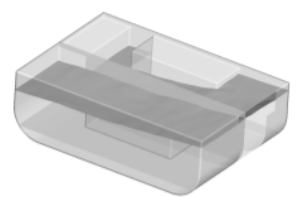

(b) 1.0 cycle

(b) damaged ship case

Fig.9 Snapshots of internal water motion at $\omega=0.415 \mathrm{rad} / \mathrm{s}$

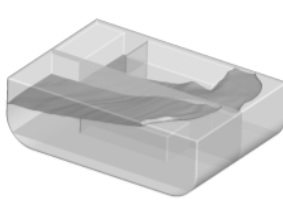

(a) 0.25 cycle

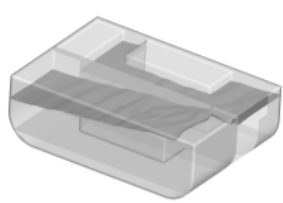

(b $) 0.25$ cycle

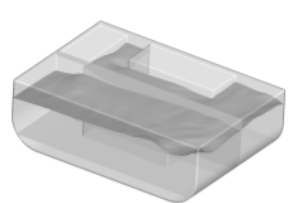

(a $) 0.5$ cycle

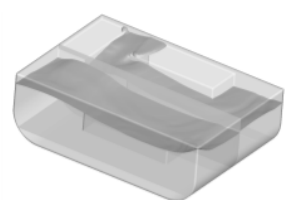

(a 1 ) 0.75 cycle

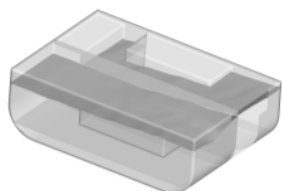

(b 1$) 0.5$ cycle

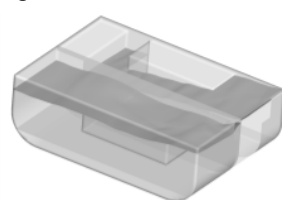

(b) 0.75 cycle

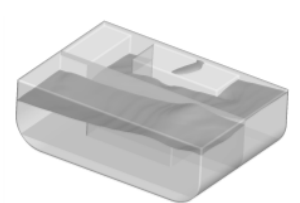

(a 1.0 cycle

(b) damaged ship case

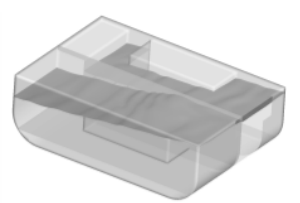

(b) 1.0 cycle

Fig. 10 Snapshots of internal water motion at $\omega=0.75 \mathrm{rad} / \mathrm{s}$ 


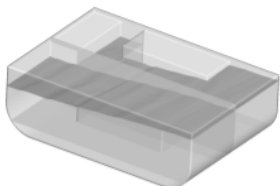

(a) 0.25 cycle

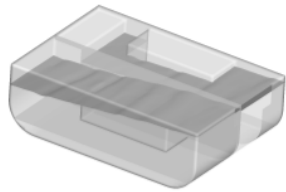

(b $) 0.25$ cycle

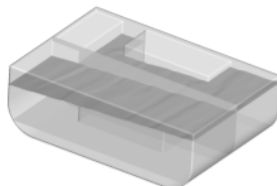

(a) 0.5 cycle

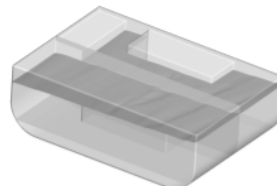

(a $a_{1} 0.75$ cycle

(a) intact ship case

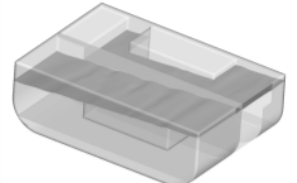

$\left(b_{1}\right) 0.5$ cycle

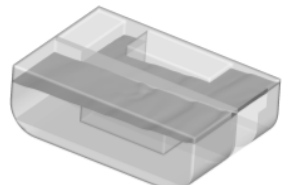

$\left(b_{1}\right) 0.75$ cycle

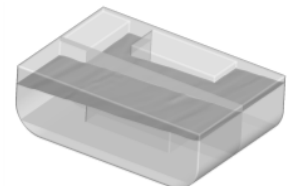

(a 1.0 cycle

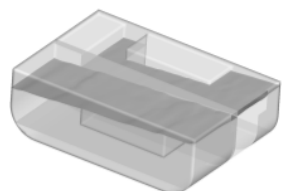

$\left(b_{1}\right) 1.0$ cycle

Fig. 11 Snapshots of internal water motion at $\omega=0.9 \mathrm{rad} / \mathrm{s}$ 